\title{
In vitro bromodeoxyuridine labelling of renal biopsy specimens: Correlation between labelling indices and tubular damage
}

\author{
K V Prasad, J Wheeler, H Robertson, W H B MaWhinney, M I McHugh, A R Morley
}

\begin{abstract}
Aims-To examine the correlation between bromodeoxyuridine (BrdU) labelling indices (LI) and tubular damage in renal biopsy specimens; to evaluate the diagnostic and prognostic potential of measuring cell proliferation in a variety of renal lesions.

Methods-In vitro BrdU labelling of renal biopsy specimens was undertaken and labelled cells were detected in routinely fixed, paraffin wax embedded sections by immunohistochemistry. The BrdU LI were calculated as percentages for the three types of tubular cellsproximal and distal convoluted tubules and medulla (LI/PCT, LI/DCT, LI/Med)-and a total tubular BrdU LI (LI/Tub) was also calculated for each biopsy specimen. Histological features indicative of tubular damage were also scored and a total tubular damage score obtained for each biopsy specimen.
\end{abstract}

Results-The one hour labelling process did not affect tissue morphology or impede subsequent diagnosis. Four biopsy specimens were obtained from three renal transplant recipients. Diagnosis of 19 non-transplant biopsy specimens revealed a variety of renal lesions. Total tubular damage scores ranged from 0 to 25 and the LI/Tub ranged from 0 to $3.68 \%$ in all 23 biopsy specimens. Analyses of variance showed highly significant correlations between the total tubular damage score and both LI/Tub ( $p=0.004)$ and LI/PCT ( $p=$ 0.004); a weaker correlation was found between the total tubular damage score and LI/DCT ( $p=0.013)$.

Conclusions-A correlation was found between tubular damage and BrdU $L I$. This was most clearly seen in the proximal tubules. However, as the study was limited to a few examples of specific forms of glomerular or interstitial disease, firm conclusions about the value of BrdU labelling in routine diagnosis and prognosis could not be drawn.

$(\Im$ Clin Pathol 1994;47:1085-1089)

Renal biopsy has a pivotal role in the diagnosis and prognosis of renal disease. Consequently, conventional methods of histopathology, immunofluorescence, and electron microscopy have remained almost unaltered for two decades. However, these are essentially "static" methods and provide little information about "dynamic" processes such as cell proliferation, which may contribute significantly to the development of certain lesions, such as crescentic glomerulonephritis and acute tubular disorders. ${ }^{1}$ To date, there have been few studies on the contribution of cell proliferation to renal disease in humans.

The estimation of cell cycle parameters based on the proportion of cells in DNA synthesis ( $\mathrm{S}$ phase), such as the labelling index $(\mathrm{LI})$, is widely used in experimental oncology, forming part of the rationale for treatment with cycle and phase specific drugs. ${ }^{2}$ Traditionally, tritiated thymidine autoradiography has been used but as this involves infusion of a long-lived, radioactive isotope, few studies have been undertaken in humans and none in patients with inflammatory renal disease. Furthermore, the difficulties of autoradiography and the several weeks required to obtain results have precluded such methods in the clinical context. A more convenient alternative to tritiated thymidine is the incorporation of the thymidine analogue bromodeoxyuridine (BrdU) into the DNA of cells in S phase. ${ }^{3}$ Cells labelled in this way can be detected rapidly with a monoclonal antibody to $\mathrm{BrdU}$ and routine immunohistochemical techniques. ${ }^{4}$

Although BrdU is not radioactive, it is a possible mutagen and teratogen and, therefore, has been administered in vivo to only a few patients with advanced malignant disease. ${ }^{5}$ However, a number of studies have successfully obtained the $S$ phase $\mathrm{LI}$ in human tissues following in vitro incubation with BrdU. These have been undertaken principally in skin, ${ }^{6}$ endometrium, ${ }^{78}$ breast, lymph node, and bone marrow ${ }^{9}$-rapidly proliferating tissues. Recent studies from this laboratory, with a mouse model of antiglomerular basement membrane glomerulonephritis, have shown that $S$ phase LI can be obtained in the slowly proliferating kidney, following either in vivo $^{1011}$ or in vitro ${ }^{12}$ labelling with BrdU.

In this paper we report the results of a new technique, in which renal biopsy specimens were labelled in vitro with BrdU before routine processing. Labelling indices in the tubules were determined and the biopsy specimens evaluated for tubular damage using conventional light microscopy. 


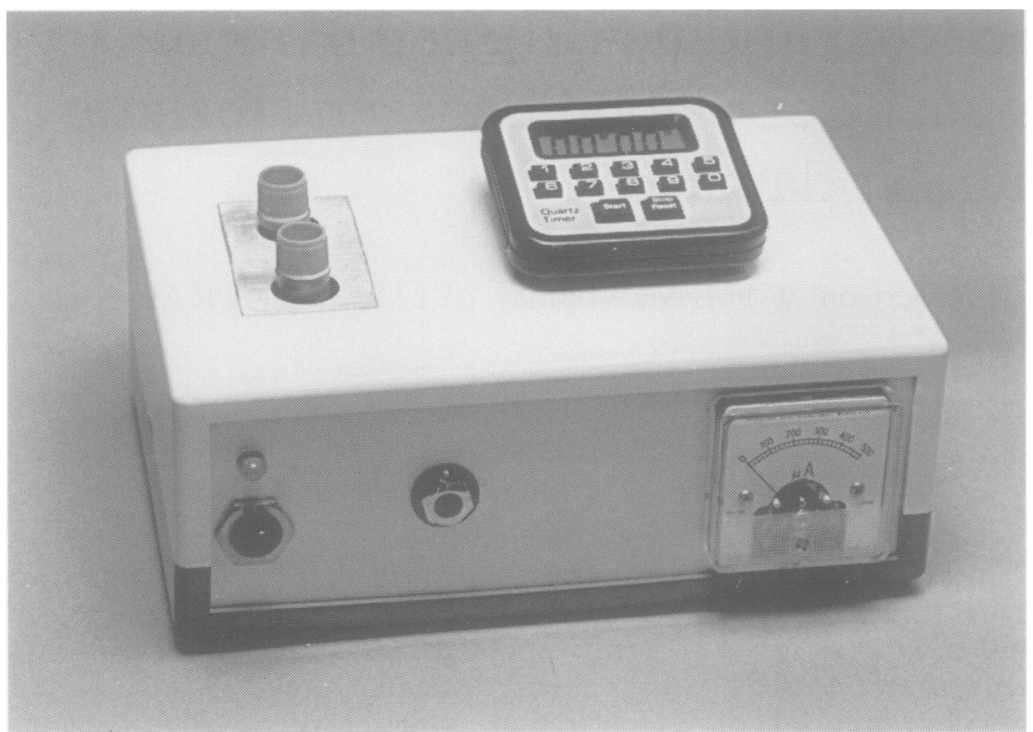

Figure 1 Portable, rechargeable incubation block, containing biopsy incubation tubes.

\section{Methods}

Needle biopsy specimens, $1.8 \mathrm{~mm}$ in diameter, were obtained from renal patients in the Royal Infirmary, Sunderland, and Freeman Hospital, Newcastle upon Tyne. All patients were undergoing biopsy for diagnostic purposes.

The labelling medium was RPMI 1640, supplemented with penicillin/streptomycin solution at $100 \mathrm{U} / \mathrm{ml} / 100 \mu \mathrm{g} / \mathrm{ml}$, HEPES buffer ( $\mathrm{pH} 7 \cdot 3$ ) at $20 \mathrm{mM}$, and L-glutamine at $2 \mathrm{mM}$. The osmolality of the medium was adjusted to $290 \mathrm{mosmol} / \mathrm{kg}$ with sterile distilled water. Aliquots $(1.93 \mathrm{ml})$ were stored for up to one week at $4^{\circ} \mathrm{C}$ in sterile, capped, plastic tubes.

Immediately before use, $10 \mu \mathrm{l}$ each of BrdU and hydrogen peroxide solutions were added to each aliquot to give final concentrations of $10 \mu \mathrm{M}$ and $0.015 \%$, respectively.

It was essential that all renal cores be incubated as soon as possible after biopsy. We therefore designed and constructed a portable, rechargeable, battery operated, aluminium incubation block (fig 1). The block was designed to require the minimum of attention from the clinician or radiologist performing the biopsy.

Table 1 Indicators of tubular damage and proliferation

\begin{tabular}{ll}
\hline Tubular changes & $\begin{array}{l}\text { Tubular loss } \\
\text { Tubular cell necrosis } \\
\text { Tubular cell mitoses } \\
\text { Tubular regeneration } \\
\text { Tubular dilatation } \\
\text { Loss of brush borders } \\
\text { Tubular casts and dead cells }\end{array}$ \\
Bowman's space & $\left\{\begin{array}{l}\text { Dilatation of Bowman's space } \\
\text { Tubularisation of basement membrane }\end{array}\right.$ \\
Interstitial changes & $\left\{\begin{array}{l}\text { Interstitial oedema } \\
\text { Interstitial inflammation } \\
\text { Interstitial fibrosis }\end{array}\right.$ \\
Other changes & $\left\{\begin{array}{l}\text { Juxta-glomerular changes } \\
\text { Intravascular neutrophils }\end{array}\right.$
\end{tabular}

The incubation block was constructed around a $25 \mathrm{~mm} \times 50 \mathrm{~mm} \times 50 \mathrm{~mm}$ aluminium block with two holes $(15 \mathrm{~mm}$ in diameter, $35 \mathrm{~mm}$ deep) in the top surface to accommodate the tubes containing the biopsy specimen in labelling medium. The temperature of the medium was maintained at $37^{\circ} \mathrm{C}$ by two resistors (4.7 $\Omega 25 \mathrm{~W})$ fixed to the block, and powered by four rechargeable cells ("D" size $1.25 \mathrm{~V}$ ). The temperature was controlled by a thermistor inserted into the centre of the block and a LM324 op-amp switching the two resistors through a TIP3055 power switching transistor. The rechargeable cells have a capacity of four amp hours giving at least two hours' use of the incubation block. In practice, the block operates satisfactorily for up to six hours between charges.

Immediately after biopsy, each renal core was placed in $1.95 \mathrm{ml}$ labelling medium containing BrdU and hydrogen peroxide, prewarmed to $37^{\circ} \mathrm{C}$ in the incubation block. Incubation was continued for one hour, during which time the block containing the specimen was taken to the histopathology laboratory.

After labelling, biopsy specimens were placed into sterile $0.01 \mathrm{M}$ phosphate buffered saline (PBS), $\mathrm{pH} 7 \cdot 2$, for routine processing and diagnosis. Samples of each biopsy core were taken for immunofluorescence and electron microscopy and the remainder was fixed in formal sublimate for 45 minutes at room temperature. After fixation, the biopsy specimens were transferred to $70 \%$ ethanol, before processing and embedding in paraffin wax $\left(56^{\circ} \mathrm{C}\right)$. Sections, at 2 to $4 \mu \mathrm{m}$, on lysine coated slides, were prepared for routine histology and for the detection of BrdU labelled cells.

Histological assessment of each biopsy specimen was made from sections stained with periodic acid Schiff (PAS) or eosin and Mayer's haematoxylin. All sections were coded and read blind. Fourteen features indicative of tubular damage (table 1 ) were scored on a normal (0) to severe (3) scale. A total tubular damage score was then obtained for each biopsy specimen by summating the 14 individual scores.

BrdU labelled cells were detected using the method of Robertson et al. ${ }^{10}$ Briefly, the method uses a rat monoclonal antibody to BrdU (Sera-Lab Ltd, Crawley Down, Sussex; code: MAS 250b) and rabbit anti-rat immunoglobulins conjugated to horseradish peroxidase (Dako Ltd, High Wycombe, Bucks; code: P162). The substrate/chromagen used to visualise the labelled cells was hydrogen peroxide/diaminobenzidine tetrahydrochloride (DAB). Sections were counterstained with PAS and Mayer's haematoxylin.

Sections were stained for BrdU and labelled/unlabelled cells were counted, at a magnification of $\times 200$, in the tubules of the cortex and the medulla. BrdU labelling indices were calculated as percentages for the proximal convoluted tubules (LI/PCT), the distal convoluted tubules (LI/DCT), and the medulla (LI/Med). A total tubular BrdU 
Table 2 Histological diagnoses, total tubular damage scores, and BrdU LIs of 23 renal biopsy specimens

\begin{tabular}{lllllll}
\hline Code & Diagnosis & Tub score & LI/PCT & LI/DCT & LI/Med & LI/Tub \\
\hline 1 & Lupus glomerulonephritis & 14 & $1 \cdot 74$ & $1 \cdot 19$ & NMP & $1 \cdot 48$ \\
2 & Ischaemia & 13 & 0 & 0 & 0 & 0 \\
3 & Amyloidosis & 17 & $2 \cdot 09$ & $0 \cdot 60$ & $4 \cdot 10$ & $1 \cdot 23$ \\
7 & IgA:MPGN & 4 & 0 & 0 & 0 & 0 \\
$8^{\star}$ & T:cellular rejection & 21 & $3 \cdot 80$ & $3 \cdot 60$ & NMP & $3 \cdot 68$ \\
9 & MPGN & 3 & $0 \cdot 04$ & $0 \cdot 05$ & 0 & $0 \cdot 03$ \\
10 & Focal CGN & 15 & $0 \cdot 60$ & $0 \cdot 70$ & NMP & $0 \cdot 60$ \\
11 & End stage renal failure & 13 & 0 & $0 \cdot 35$ & NMP & $0 \cdot 21$ \\
12 & IgG:MPGN & 7 & $0 \cdot 06$ & 0 & 0 & $0 \cdot 02$ \\
15 & MPGN & 1 & 0 & $0 \cdot 04$ & 0 & $0 \cdot 01$ \\
16 & MGN & 4 & $0 \cdot 22$ & $0 \cdot 05$ & $0 \cdot 10$ & $0 \cdot 13$ \\
17 & MPGN & 1 & $0 \cdot 22$ & $0 \cdot 07$ & $0 \cdot 12$ & $0 \cdot 13$ \\
18 & Myeloma & 25 & $1 \cdot 57$ & $0 \cdot 70$ & NMP & $1 \cdot 29$ \\
19 & MGN & 1 & $0 \cdot 70$ & $0 \cdot 18$ & NMP & $0 \cdot 42$ \\
20 & MGN & 0 & 0 & 0 & 0 & NMP \\
21 & DPGN & 17 & 0 & 0 & 0 & 0 \\
22 & Diabetic GSc & 5 & 0 & 0 & 0 & 0 \\
23 & FSGN & 23 & $0 \cdot 04$ & $0 \cdot 20$ & NMP & $0 \cdot 11$ \\
24 & Ischaemia & 6 & $0 \cdot 07$ & $0 \cdot 18$ & $0 \cdot 17$ & $0 \cdot 14$ \\
25 & T:arteriosclerosis & 20 & $2 \cdot 33$ & $0 \cdot 84$ & $0 \cdot 46$ & $1 \cdot 26$ \\
$26^{\star}$ & T:post cellular rejection & 10 & $1 \cdot 26$ & $1 \cdot 14$ & $0 \cdot 29$ & $0 \cdot 91$ \\
27 & T:renal artery stenosis & 10 & 0.03 & $0 \cdot 13$ & 0 & $0 \cdot 03$ \\
28 & MGN & 5 & $0 \cdot 08$ & $0 \cdot 10$ & 0 & $0 \cdot 06$ \\
\hline
\end{tabular}

${ }^{\star}$ Consecutive biopsy specimens from same patient; CGN = crescentic glomerulonephritis; DPGN = diffuse proliferative glomerulonephritis; FSGN = focal segmental glomerulonephritis; GSc = glomerulosclerosis; MGN = membranous glomerulonephritis; MPGN = mesangial proliferative glomerulonephritis; NMP = no medulla present; $T$ = transplant.

labelling index (LI/Tub) was also calculated for each biopsy specimen by summating the counts from the three types of cells. All measurements were determined with coded slides.

Analysis of variance was used as detailed.

\section{Results}

Twenty three renal biopsy specimens from 22 patients were labelled in vitro with $\mathrm{BrdU}$ before routine processing. The one hour labelling process did not affect tissue mor-

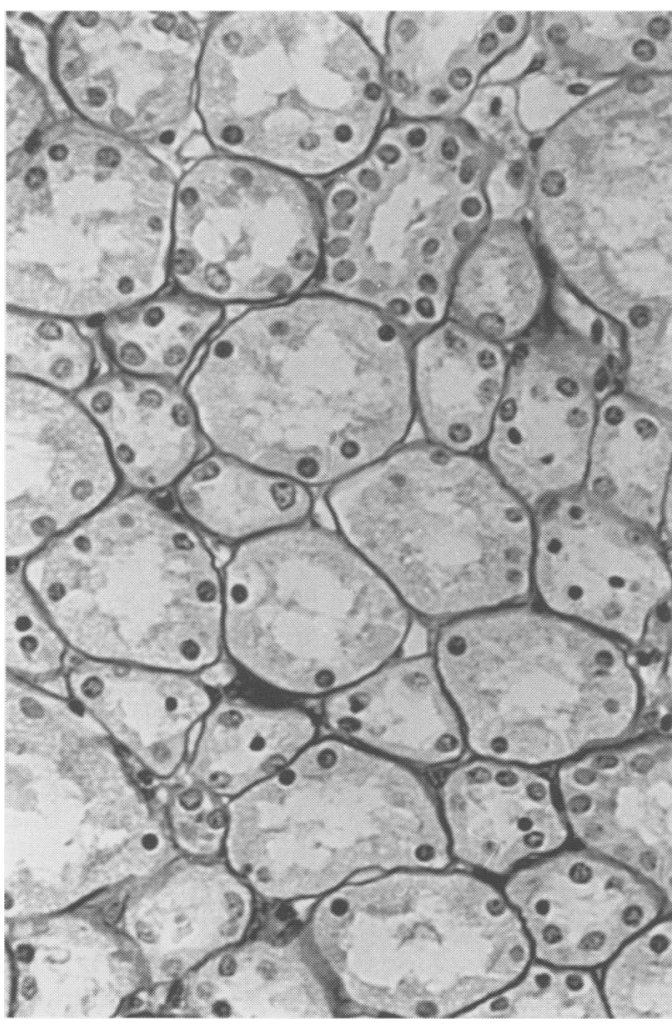

Figure 2 Membranous glomerulonephritis, showing normal tubules. Total tubular damage score $=0$ (PAS and haematoxylin). phology in any of the biopsy specimens and, hence, did not impede subsequent diagnosis of the renal lesion.

The histological diagnoses of the 23 biopsy specimens are given in table 2 , together with the total tubular damage scores and the BrdU LIs. Eight of the specimens did not contain any medulla.

Four biopsy specimens (cases 8, 25, 26, 27) were obtained from three renal transplant recipients. Two consecutive biopsy specimens were obtained from one transplant recipient: the first (case 8) was taken during a rejection episode; the second (case 26) was obtained after treatment for the rejection. The two remaining transplant biopsy specimens displayed ischaemic damage (cases 25 and 27). Diagnosis of the 19 non-transplant biopsy specimens revealed a variety of renal lesions, including several glomerulonephritides, end stage renal failure, ischaemia, amyloidosis, diabetic glomerulosclerosis, and multiple myeloma.

Total tubular damage scores ranged from 0 , in a case of membranous glomerulonephritis (case 20; fig 2), to 25 in a patient with multiple myeloma (case 18; fig 3). Usually, low tubular damage scores (1-7) were found in patients with membranous and mesangial proliferative glomerulonephritis.

$\mathrm{LI} / \mathrm{Tub}$ ranged from 0 in patients with ischaemia (case 2), diabetic glomerulosclerosis (case 22), mesangial proliferative (case 7), membranous (case 20), and diffuse proliferative glomerulonephritis (case 21 ) to $3.68 \%$ in a transplanted kidney undergoing cellular rejection (case 8; fig 4).

Analyses of variance showed highly significant correlations between the total tubular damage score and both $\mathrm{LI} / \mathrm{Tub}$ (fig $5 \mathrm{~A} ; \mathrm{p}=$ 0.004 ) and LI/PCT (fig 5B; $p=0.004$ ). A weaker but none the less significant correlation was also found between the total tubular damage score and LI/DCT (fig $5 \mathrm{C} ; \mathrm{p}=$ $0.013)$. 
Figure 3 Myeloma, showing tubular atrophy and interstitial fibrosis.

Total tubular damage score $=25$ (PAS and haematoxylin).
Figure 4 BrdU labelled tubular cells (arrows) in a transplanted kidney undergoing rejection $(L I=3.68 \%)$, showing interstitial oedema and inflammatory cell infiltrate (counterstained with PAS and haematoxylin).
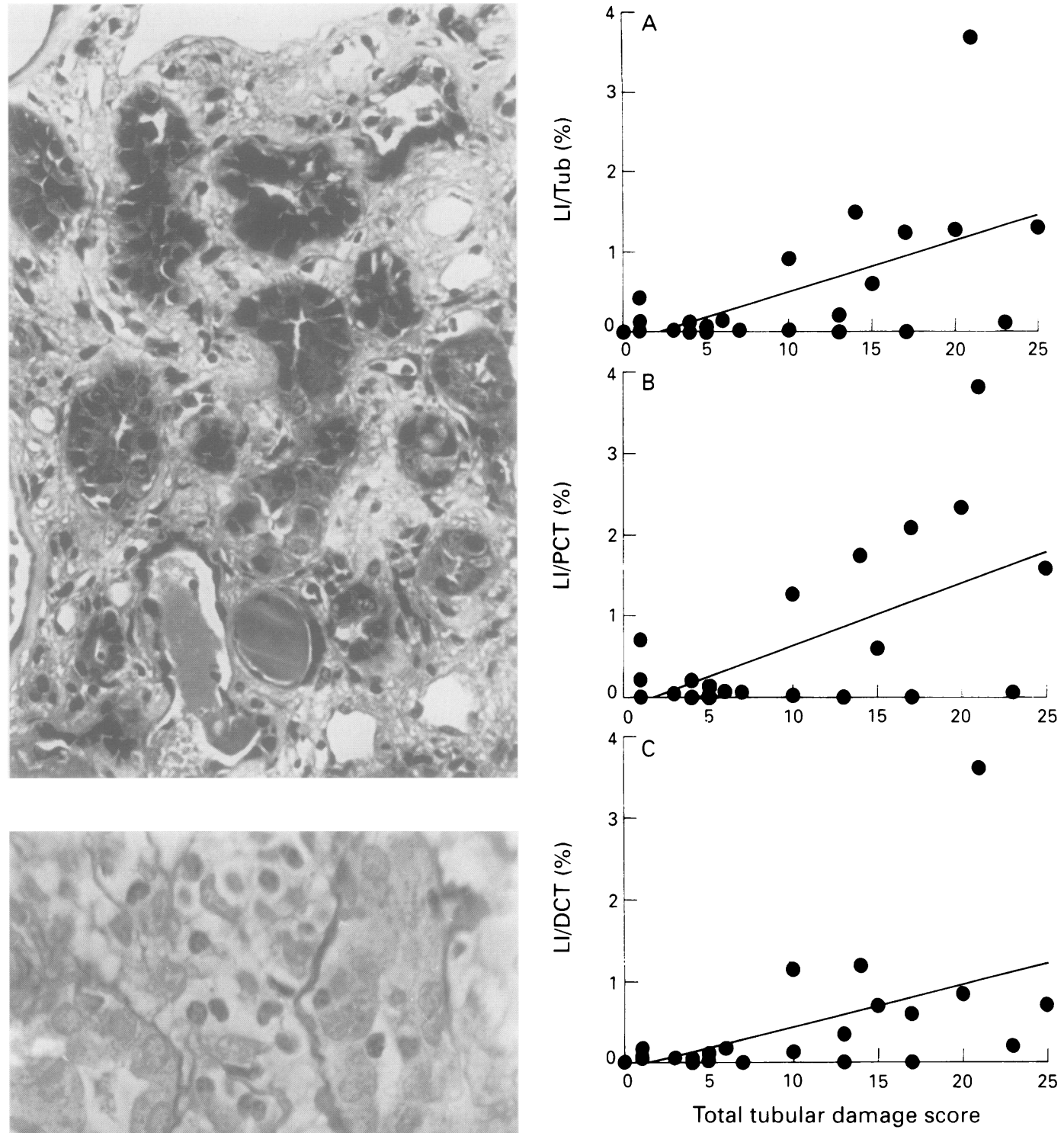

Figure 5 Relation between total tubular damage scores and $(A)$ total tubular BrdU-LI (LI/Tub); (B) proximal convoluted tubular BrdU-LI (LI/PCT); (C) distal convoluted tubular BrdU-LI (LI/DCT).

hours compared with the one hour of mitosis, many more proliferating cells will be recognised if DNA synthesising cells are labelled.

Our previous studies have shown that mouse renal tissue can be successfully cultured without loss of histological detail and with good demonstration of BrdU labelled cells. ${ }^{12}$ The preservation of histological detail is important as human renal biopsy specimens are taken only for good diagnostic reasons, are rarely repeated, and have a small element of risk for the patient. Techniques to obtain further information from the limited material available must not diminish the pathologist's ability to reach a diagnosis. It is also unlikely that a technically difficult procedure will be used by busy clinicians.

The method we have devised in which the biopsy specimen is placed in a prewarmed tissue culture medium in an incubation block has proved convenient for both clinicians and technical staff and we have observed no changes in the immunofluorescent or electron tubulointerstitial nephritides, which in transplant rejection. As renal tissue normally has a minimal mitotic rate, ${ }^{13}$ these observations are significant but difficult to interpret. Because DNA synthesis lasts about seven 
microscopic appearances. The incubation block is undergoing further development to improve temperature display.

The demonstration of BrdU labelled cells is clear, and equivocal labelling is rare. In some samples there is a suggestion that BrdU labelling occurs more often in the outer layers of tissue. This may be related to the rate of diffusion of BrdU into the tissue or possibly a change in the rate of uptake at deeper levels. Practically, it is not possible to incubate for more than one hour without affecting the diagnostic process.

The series contains too few examples of specific forms of glomerular or interstitial disease to draw general conclusions about BrdU labelling. However, there is a clear association between tubular damage and BrdU LIs, which is most clearly seen in the proximal tubules. BrdU labelling in the medulla was lower than in the cortex, but the fact that some eight out of 23 cases did not have medulla in this sample makes this observation of limited value.

Interestingly, the highest labelling was seen in a case of acute transplant rejection, and a subsequent biopsy specimen after treatment showed a considerably lower value. Consequently, further studies are concentrating specifically on BrdU labelling in renal transplant rejection. It has been agreed that invasion of tubules by lymphoid cells (tubulitis) is a better indicator of acute rejection than simple interstitial accumulation, ${ }^{14}$ although any differences in the activity of these lymphoid cells have yet to be identified. Cell proliferation with BrdU, together with phenotype studies, may clarify this issue, as the combined technique will distinguish actively rejecting populations from "inert" lymphoid cell collections.
The incubation block is now being marketed by Novocastra (Newcastle) Ltd.

1 Dunnill MS. Classification of glomerular disease. In Pathological basis of renal disease. London: Baillièr Tindall, 1984:1-10.

2 Tannock I. Cell kinetics and chemotherapy: a critica review. Cancer Treat Rep 1978;62:117-33.

3 Gratzner HG. Monoclonal antibody to 5-bromo- and 5 -iododeoxyuridine: a new reagent for detection of DNA replication. Science 1982;218:474-5.

4 Sugihara H, Hattori T, Fukuda M. Immunohistochemical detection of bromodeoxyuridine in formalin-fixed tissues. Histochemistry 1986;85:193-5.

5 Danova M, Riccardi A, Brugnatelli S, Fiocca R, Girino M Villani $L$, et al. In vivo bromodeoxyuridine incorporation in human gastric cancer: a study on formalin-fixed paraffin-embedded sections. Histochem $\mathcal{F}$ 1989;20:125-30.

6 Wolff $\mathrm{HH}, \mathrm{Gnas}$ W. Immunocytochemical detection of in vitro incorporated 5-bromodeoxyuridine in paraffin sections of human skin. Arch Dermatol Res 1989;281: 209-12.

7 Thornton JG, Wells M, Hume WJ. Flash labelling of S phase cells in short term culture of normal and patholog phase cells in short term culture of normal and pathological human endometrium using bromodeoxy

8 Thornton JG, Wells M, Hume WJ. Measurement of the S phase duration in normal and abnormal human endometrium by in vitro double labelling with bromodeoxyuridine and tritiated thymidine. F Pathol 1989;157 109-15.

9 Veronese S, Gambacorta M, Falini B. In situ demonstration of tissue proliferative activity using anti-bromodeoxyuridine monoclonal antibody. F Clin Pathol 1989; 42:820-6.

10 Robertson $\mathrm{H}$, Wheeler J, Morley AR. In vivo bromodeoxyuridine incorporation in normal mouse kidney: immunohistochemical detection and measurement of labelling indices. Histochem $\mathcal{f}$ 1990;22:209-14.

11 Wheeler J, Robertson H, Morley AR, Appleton DR. Antiglomerular basement membrane glomerulonephritis (anti-GBM GN) in the mouse: BrdU-labelling indices and histological damage. Int $\mathcal{E}$ Exp Pathol 1992;74:9-19.

12 Robertson $\mathrm{H}$, Wheeler J, Morley AR Culture and in vitro bromodeoxyuridine-labelling of mouse kidney explants. F Tissue Culture Methods (in press).

13 Wilson GD, Soranson JA, Lewis AA. Cell kinetics of mouse kidney using bromodeoxyuridine incorporation and flow cytometry: preparation and staining. Cell Tissue and flow cytometry: prep

14 Solez K, Axelsen RA, Benediktsson H, Burdick JF, Cohen $\mathrm{AH}$, Colvin RB, et al. International standardization of criteria for the histologic diagnosis of renal allograft rejection: The Banff working classification of kidney transplant pathology. Kidney Int 1993;44:411-22. 